\title{
Developing a questionnaire to measure patients' beliefs about inhaler treatment: a pilot study
}

C H Hand

\begin{abstract}
Objective: To develop a valid and reliable questionnaire to assess patients' beliefs about inhaler treatment for asthma.

Design: A structured interview in which patients' beliefs about inhalers were individually rated. Factor analysis of those beliefs that had the highest correlations with estimated inhaler use.

Setting and subjects: A stratified random sample of 40 patients taking a combination of salbutamol and beclomethasone dipropionate in one general practice. Results: Six factors explained $67.7 \%$ of the variance relating to beclomethasone dipropionate use: positive beliefs about the inhaler, satisfaction with the repeat prescription system, dissatisfaction with the doctor, collecting inhalers, a preference for tablets and concern about side-effects. Seven factors explained $69.9 \%$ of the variance relating to salbutamol use: no faith in prevention, a dislike of inhalers, relying on regular salbutamol, lack of disability, a preference for tablets, making decisions about inhalers and inhalers not lasting long enough.

Conclusions: Beliefs about inhaler treatment can be classified into relatively few factors that explain a significant proportion of the beliefs that relate to inhaler use. Development of the questionnaire is continuing to produce a valid questionnaire with reliable subscales.
\end{abstract}

\section{INTRODUCTION}

Adherence to treatment regimens in asthma remains a problem; over use of reliever inhalers and under use of inhalers for prevention is common. ${ }^{1}$ The concept of adherence has moved away from the view of the passive patient complying with medical advice, to that of the patient actively making decisions with the doctor to improve their health. Patients' ideas about their illness and its treatment are clearly important in this negotiation. ${ }^{2}$

In the psychological field, social cognitive and selfregulatory models have been used to explain and predict health behaviours, including adherence. ${ }^{1,3}$ Relatively little has been done with asthma compared to other chronic diseases such as diabetes. Qualitative research has provided some insight. Issues such as the difficulty of assessing risks and benefits of preventive treatment, stigma, concern about side-effects and fear of dependency are common themes. ${ }^{4.5}$ Quantifying the issues has been less well researched, and few tools exist to identify those at risk of poor adherence in general practice. A set of questions that could link what patients think about their inhaler treatment to how they use inhalers would be helpful not only for identifying suboptimal use, but also for researching ways of improving adherence. We know that asking patients directly about adherence is associated with improved adherence, but what questions should be asked? ${ }^{6}$ This study aims to develop a valid and reliable questionnaire to measure patients' beliefs about inhaler treatment for asthma.

\section{METHODS}

\section{Patient selection}

A list of patients taking a combination of salbutamol and beclomethasone dipropionate inhalers was obtained by computer search in the author's practice of 10,000 patients on the borders of Norfolk and Suffolk. A stratified sample was chosen to ensure a wide age range (17-83, median 56 years), equal numbers of men (20) and women (20) and patients representing each of the six general practitioners. The diagnosis of asthma was verified by either objective evidence from peak expiratory flow (PEF) readings (32 patients) or subjective evidence of cough and wheeze that responded to inhaler treatment (eight patients). Half of the patients had asthma for over 16 years (2-80, median 17.5 years). The patients were contacted by telephone; all agreed to participate. The structured interviews were conducted in the patients' homes by the author.

\section{Development of the structured interview}

Semi-structured interviews with eight patients were analysed qualitatively and eight main themes were identified. Patients' views were allocated to each theme, resulting in a structured interview of 102 statements, 92 of which covered beliefs about inhaler treatment.

Interview procedure and estimation of inhaler use The statements were read out from the interview schedule and the extent to which the patient agreed or disagreed was recorded on a five-point Likert scale (strongly agree to strongly disagree). The number of salbutamol and beclomethasone dipropionate inhalers ordered in the previous year was counted from both the computer and written records. Inhaler use was defined as the average number of puffs per day based on these figures.

\section{Statistical analysis}

The data were entered into the Statistical Package for Social Sciences. Spearman's rank correlations were calculated using the rating scores of the individual statements and the number of puffs per day of each inhaler. Statements were selected for entry into the factor analysis in order of the strength of their correlation with inhaler use until 10 positive and 10 negative statements were obtained. As there were 15 statements that had significant $(\mathrm{p}<0.01)$ positive correlations with beclomethasone dipropionate use, all of these were used.

Factor analysis was performed in two steps; one for each inhaler. Principal component analysis was used with Varimax rotation and Kaiser normalisation. Factors with Eigenvalues greater than one were selected. Spearman's rank correlations were calculated using all the identified factors. The internal reliabilities of each multi-item beclomethasone dipropionate and salbutamol scale were tested using Cronbach's alpha co-efficient.

\section{RESULTS}

All of the statements were answered by all of the participants. A satisfactory spread of responses was seen, with only two items having markedly skewed responses. Two patients disagreed with the statement, 'I prefer my salbutamol inhaler to my beclomethasone inhaler' and nobody disagreed with, 'Preventing an 
asthma attack is better than waiting for one to happen'.

Six factors explained $67.7 \%$ of the variance relating to beclomethasone dipropionate use and for salbutamol use seven factors explained $69.9 \%$. The statements, their factor loadings and variance of each factor are shown in Table 1 (beclomethasone dipropionate) and Table 2 (salbutamol). Table 3 shows the correlations between each of the scales. The Cronbach's alpha coefficients for the multi-item scales were between 0.28 and 0.92 (Tables 1 and 2).

\section{DISCUSSION}

Several of the beliefs and relationships identified in this study have been found by others. The influence of patient satisfaction on compliance has been known for some time. ${ }^{8}$ Concern about the side-effects of steroids, fears of dependency, and embarrassment using inhalers in public are all well documented. ${ }^{4,59}$ Although a dislike of asthma medication can exist independently of whether the medication is for prevention or relief, not all surveys distinguish between inhalers. ${ }^{10,11}$

The factors identified in this study account for over two thirds of the variance relating to the estimated use of beclomethasone dipropionate and salbutamol inhalers. A factor loading greater than 0.45 is an acceptable correlation with a factor and, using this criterion, only two items loaded onto more than one factor. ${ }^{12}$ Whilst the interpretation of factors is subjective, some of those that emerged in this analysis have face validity and the potential for further development. The generally low inter-scale correlations demonstrate that the scales appear to measure different issues relating to adherence.
Table 1: Beliefs relating to beclomethasone use, with factor correlations of greater than $\mathbf{0 . 3 2}$ (with correlations below 0.45 in brackets) and percentage of variance explained by each factor.

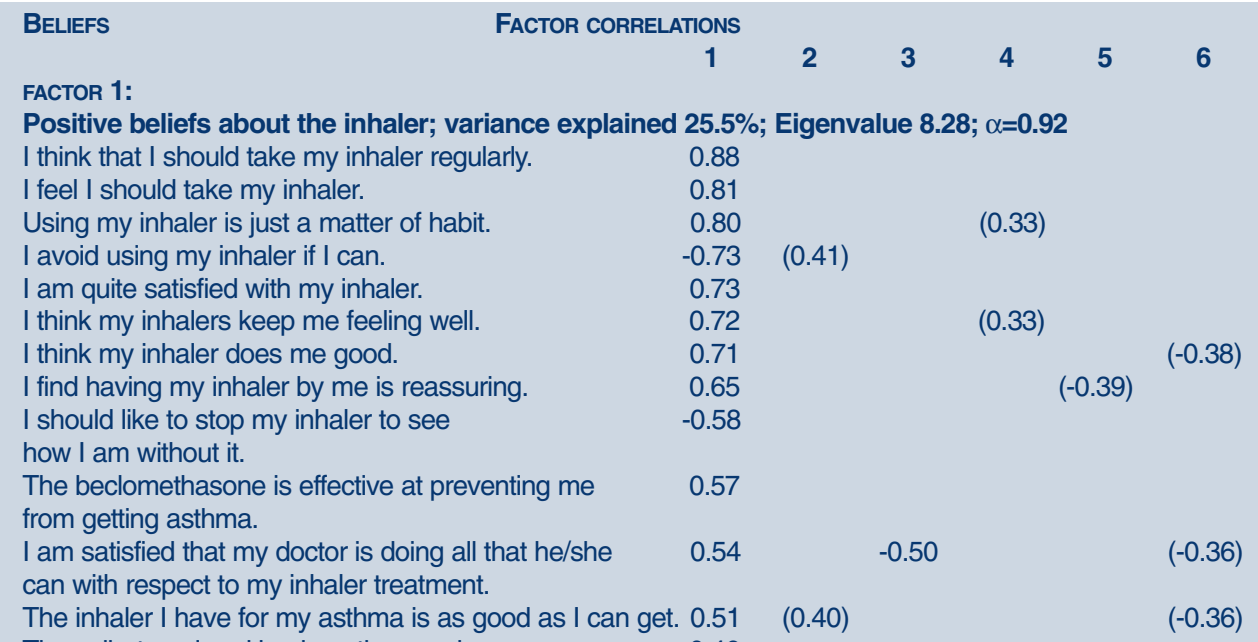

The salbutamol and beclomethasone have $\quad 0.46$

to be taken together to work properly.

FACTOR 2:

Satisfaction with the repeat prescription system; variance explained $9.1 \%$; Eigenvalue $2.64 ; \alpha=0.54$ I am quite satisfied with the system for getting $\quad 0.74$

repeat prescriptions of my inhalers.

The system for getting repeat prescriptions $\quad-0.69$

of my inhalers is inconvenient.

It should be possible to get my inhalers delivered 0.51

to my house if I can't get them myself.

FACTOR 3:

Dissatisfaction with the doctor; variance explained $8.9 \%$; Eigenvalue $1.80 ; \alpha=0.71$

$\begin{array}{ll}\text { There is never enough time to talk about how I feel } & 0.79\end{array}$

about my inhaler treatment.

Sometimes I think my doctor doesn't tell me

the whole truth about my inhaler.

FACTOR 4:

Collecting inhalers; variance explained 8.5\%; Eigenvalue 1.77; $\alpha=0.77$

I prefer to collect my repeat prescriptions on a

regular basis, then I know where I am.

I usually plan ahead to get my inhalers

FACTOR 5:

Preference for tablets; variance explained 8.1\%; Eigenvalue 1.25; $\alpha=0.57$

I would prefer to take a tablet than use an inhaler.

I avoid using an inhaler in public.

Taking inhalers is inconvenient.

$(-0.38)$

FACTOR 6:

Concern about side effects; variance explained 7.6\%; Eigenvalue 1.18; $\alpha=0.47$

I am worried about possible long-term side effects

(0.33) $\quad 0.80$

from my inhalers.

I think I should use my inhaler as little as possible.

Given that a value of 0.7 for Cronbach's alpha is usually taken as being acceptable for a scale, the reliability of three of the subscales was satisfactory, three were borderline and seven were poor (less than 0.6 ). Work is in progress to improve the reliability by adding additional items to the scales in a study with a larger number of subjects.

\section{Beliefs relating to beclomethasone use (Table 1)}

The first factor contains the positive beliefs that one would expect to underpin adherence to a preventive treatment regimen. The second and fourth factors relate to satisfaction with the practice's system of supplying further supplies of treatment and to personal organisation in obtaining inhalers. The third factor clearly represents dissatisfaction with the doctor. Factor five points to a preference for taking tablets, and factor six relates to concerns about sideeffects.

\section{Beliefs relating to salbutamol use (Table 2)}

The interpretation of some of the salbutamol factors is not straightforward and these need further development. The first factor indicates that the patient has little faith in prevention with beclomethasone dipropionate, perhaps 'explaining' the third factor (relying on regular salbutamol). Factor five relates to a preference for tablets, factor six to making decisions about inhalers and factor seven to inhalers not lasting long enough. Factor two appears to indicate a dislike of inhalers, whilst factor four points to a relationship between lack of disability and the effects of steroid inhalers.

\section{Relationships between the factors (Table 3)}

There were only five significant correlations between the factors and two of these were at the $5 \%$ level of significance. Given that 78 correlations were performed, these two correlations may have occurred 
Table 2: Beliefs relating to salbutamol use, with factor correlations of greater than 0.32 (with correlations below 0.45 in brackets), percentage of variance explained by each factor, Eigenvalues and alpha co-efficients

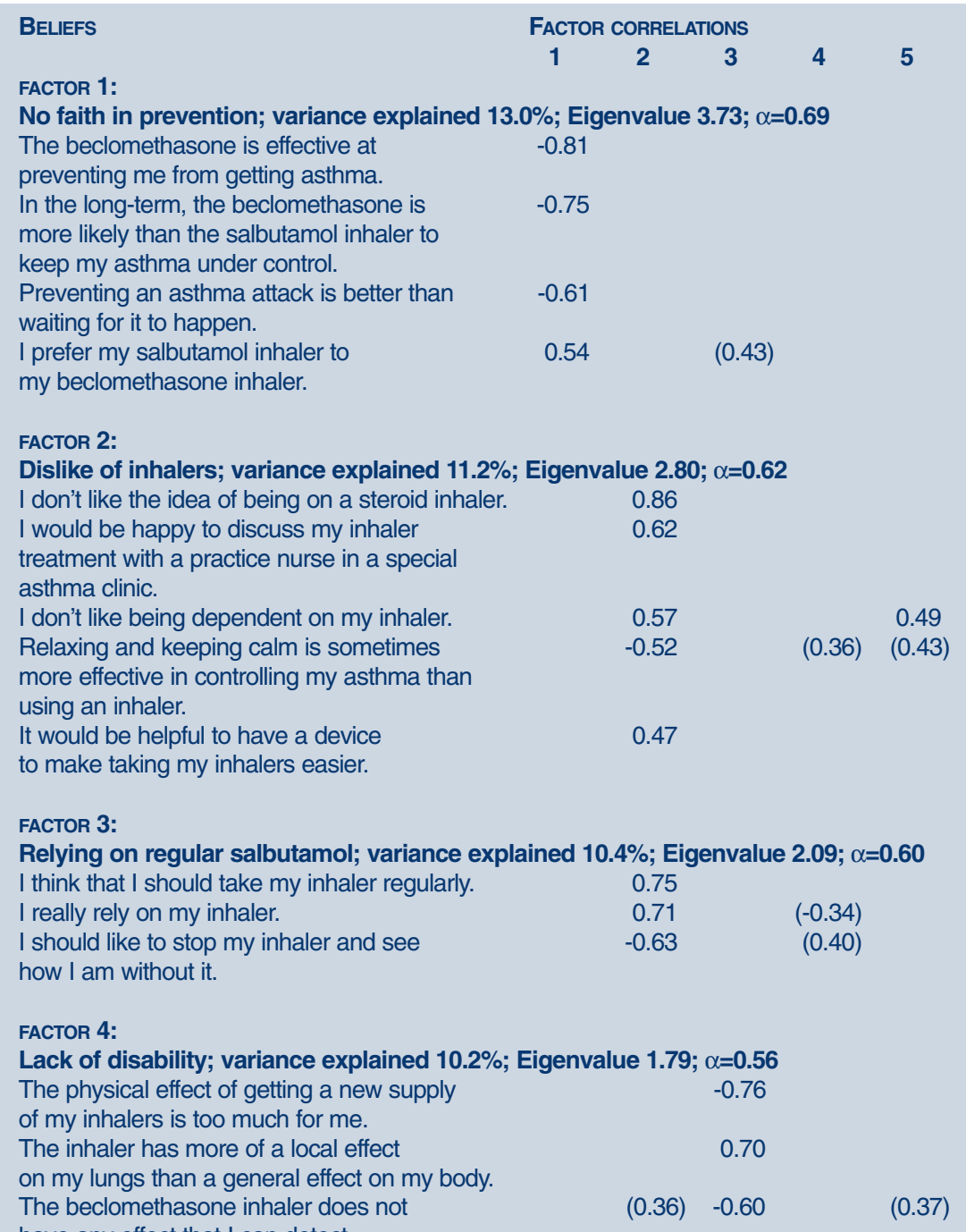

FACTOR 5:

Preferences for tablets; variance explained 9.5\%; Eigenvalue 1.41; $\alpha=$ not applicable I would prefer to take a tablet than use an inhaler.

FACTOR 6:

Making decisions about inhalers; variance explained 9.0\%; Eigenvalue 1.12; $\alpha=0.49$ I would be interested to try a new inhaler

to see if it is any better for my asthma.

The decision when to use my inhaler is

mine alone.

FACTOR 7:

Inhalers not lasting long enough; variance explained $6.6 \%$; Eigenvalue $1.04 ; \alpha=0.28$ I find it difficult to know when my inhalers

are running out.

The effect of the inhaler doesn't last long enough.

(0.35) $\quad(-0.42)$

\section{Limitations of the study}

There are limitations to this study. First, it was performed in one practice where the author was known to most of the patients. However, patients did make some strong criticisms in the semistructured interviews and in the structured interviews there was a good spread of responses in all bar two of the items. It is important to know whether these views are more widely held and this is currently being tested in a number of practices.

The second criticism is that the relationships between a large number of variables were explored in a small sample of patients. In factor analysis there should be four times as many variables as subjects and as a general rule one should have at least 300 subjects. The analysis should, therefore, be considered exploratory rather than definitive. The method of selecting variables has some logic to it; i.e. choosing beliefs that have higher correlations with inhaler use, although not all the correlations were statistically significant.

Inhaler use is notoriously difficult to determine and even measuring use by incorporating computer devices has its problems. ${ }^{1}$ The method chosen has the advantage of simplicity and is widely used, but relies on the premise that if someone orders an inhaler they intend to use it. Those ordering more inhalers are probably using their inhalers more, and rank correlations reflect this.

Finally, some of the older patients probably had chronic obstructive pulmonary disease as well as asthma, and their beliefs may well be different to those who just have asthma.

\section{Future development}

The factors that have emerged in this study need refining and the subscales developed by incorporating additional items to improve reliability. Further qualitative work is needed to define additional concepts to explain more of the variance of patient behaviour. Development of the questionnaire will provide an instrument that can be used not only to estimate adherence to treatment, but also to evaluate the

by chance. The highest negative correlation was between the first beclomethasone dipropionate factor (positive beliefs) and the first salbutamol factor (no faith in prevention) and this is perfectly reasonable. The high correlation between both factor fives (tablet preference) was predictable. The negative correlation between beclomethasone dipropionate factor six (concern about side-effects) and salbutamol factor four, which contains beliefs about beclomethasone dipropionate having either a local or no discernable effect, is also understandable.

\section{ACKNOWLEDGEMENTS}

I thank Clare Bradley who supervised my MSc project in the Department of General Practice, UMDS, London. I also thank the partners and patients of the Bungay practice for their support and my colleagues in the School of Health Policy and Practice at UEA for helpful advice. Thanks are also due to the anonymous referees for their comments and to Robert McKinley for 
helping me revise the paper. The Norfolk and Norwich Bicentenary Trust kindly provided a grant.

\section{References}

1. Hand C. Adherence and asthma. In: Myers L B and Midence K, eds. Adherence to treatment in medical conditions. Amsterdam, Harwood Academic Press, 1998; 383-421.

2. Britten N. Patients' ideas about medicines: a qualitative study in a general practice population. $\mathrm{Br}$ J Gen Pract 1994; 44: 465-8.

3. Conner M, Norman P, eds. Predicting health behaviour. Buckingham, Open University Press, 1996.

4. Hibbert D. Professional and user perspectives on asthma care and inhaler therapy [ $\mathrm{PhD}$ thesis] Manchester, Manchester University Press, 1997. 5. Kibble K T. A qualitative study of patients attitudes to their asthma and its treatments. Asthma in Gen Pract 1997; 5(2): 25-6.

6. Hall J A, Roter D L, Katz N R. Meta-analysis of correlates of provider behavior in medical encounters. Medical Care 1988; 26: 657-75.

7. Hand C H, Bradley C. Health beliefs of adults with asthma: toward an understanding of the difference between symptomatic and preventive use of inhaler treatment. J of Asthma 1996; 33: 331-8.

8. Ley P. Satisfaction, compliance and

communication. Br J Clin Psychol 1982; 21: 241-54. 9. Barritt PW, Staples E B. Measuring success in asthma care: a repeat audit. Br J Gen Pract 1991; 41: 232-6.

10. Osman L M, Russell I T, Friend J A R et al. Predicting patient attitudes to asthma medication. Thorax 1993; 48: 827-30.

11. National Asthma Campaign. The impact of asthma. London, NAC, 1996.
Table 3: Correlations between beclomethasone dipropionate and salbutamol factors (Spearman's rho) with two-tailed significance levels

\begin{tabular}{|c|c|c|c|c|c|c|c|c|c|c|c|c|c|}
\hline & S1 & S2 & S3 & S4 & S5 & S6 & S7 & B1 & B2 & B3 & B4 & B5 & B6 \\
\hline S1 & 1.000 & & & & & & & & & & & & \\
\hline S2 & 0.071 & 1.000 & & & & & & & & & & & \\
\hline S3 & 0.033 & 0.025 & 1.000 & & & & & & & & & & \\
\hline S4 & 0.033 & -0.113 & 0.083 & 1.000 & & & & & & & & & \\
\hline S5 & 0.087 & 0.045 & 0.022 & -0.060 & 1.000 & & & & & & & & \\
\hline S6 & 0.022 & -0.004 & 0.048 & 0.031 & 0.019 & 1.000 & & & & & & & \\
\hline S7 & -0.43 & -0.13 & 0.013 & 0.002 & -0.059 & 0.067 & 1.000 & & & & & & \\
\hline B1 & $-0.652^{\star *}$ & -0.47 & 0.123 & -0.017 & -0.188 & -0.264 & 0.029 & 1.000 & & & & & \\
\hline B2 & 0.019 & 0.182 & 0.163 & 0.190 & -0.057 & -0.016 & 0.107 & 0.030 & 1.000 & & & & \\
\hline B3 & 0.085 & $0.324^{*}$ & -0.127 & 0.168 & -0.076 & 0.096 & -0.020 & 0.144 & -0.055 & 1.000 & & & \\
\hline B4 & 0.067 & 0.061 & 0.164 & -0.094 & 0.141 & -0.294 & -0.163 & 0.062 & 0.060 & 0.073 & 1.000 & & \\
\hline B5 & 0.122 & 0.211 & $-0.379^{*}$ & -0.168 & $0.537^{\star \star}$ & 0.072 & 0.245 & -0.110 & 0.107 & -0.084 & -0.232 & 1.000 & \\
\hline B6 & 0.088 & 0.213 & -0.244 & $-0.489^{\star \star}$ & ${ }^{*}-0.131$ & 0.161 & -0.083 & -0.083 & 0.105 & -0.048 & -0.040 & 0.094 & 1.00 \\
\hline & $\begin{array}{l}0.001 \\
0.05\end{array}$ & & & & & & & & & & & & \\
\hline
\end{tabular}

\section{Summary of statistical terms}

- Spearman's correlation co-efficient (rho): a measure of association between two variables that are not normally distributed (non-parametric test)

- Cronbach's alpha: a measure of internal reliability or consistency of a multiple-item scale that relies upon the associations of each item with each other (inter-item correlation).

- Factor analysis: a complex statistical technique used to identify a relatively small number of factors that can be used to represent relationships among sets of many interrelated variables. It is usually done in four steps:

- Correlation: variables that do not appear related to other variables are identified.

- Factor extraction: the number of factors needed to represent the data is determined.

- Rotation: the factors are transformed to make them more interpretable.

- Factor scoring: scores for each factor are computed for each case.

\title{
Concerns and misconceptions regarding steroid therapy in asthma: findings and impact of a public meeting
}

\author{
D Price, J Hobbs, S Watkins, M Duerden and H Darby
}

\section{SUMMARY}

Anxiety concerning long-term steroid therapy may be translated into non-compliance with prescribed asthma treatment; this was addressed at a public meeting. Questionnaire responses indicated an immediate, positive impact on participants' attitudes to, and misconceptions of, anti-asthma steroid therapy.

\section{INTRODUCTION}

Patient non-compliance is one factor limiting the efficacy of inhaled steroids in asthma management. ${ }^{1}$ Whilst many factors can contribute to patients' nonadherence to therapy, one key element is anxiety about steroid side-effects. ${ }^{2}$ Media reports constantly fuel fears and patients often present with 'scare stories'. Following one particular television programme some patients in a Norwich practice reduced, and others even suspended, steroid therapy.
12. Tabachnick B G, Fidell L S. Using multivariate statistics (3rd edition). New York, HarperCollins, 1996. 